\author{
Christopher W. Johnson and Gregg T. Beckham* \\ National Bioenergy Center, National Renewable Energy Laboratory, Golden CO 80401 \\ *Corresponding Author Information: gregg.beckham@nrel.gov; +01-303-384-7806
}

KEYWORDS: lignin valorization, $\beta$-ketoadipate pathway, aromatic degradation, Pseudomonas putida KT2440, Sphingobium sp. strain SYK-6

\begin{abstract}
Lignin represents an untapped feedstock for the production of fuels and chemicals, but its intrinsic heterogeneity makes lignin valorization a significant challenge. In nature, many aerobic organisms degrade lignin-derived aromatic molecules through conserved central intermediates including catechol and protocatechuate. Harnessing this microbial approach offers potential for lignin upgrading in modern biorefineries, but significant technical development is needed to achieve this end. Catechol and protocatechuate are subjected to aromatic ring cleavage by dioxygenase enzymes that, depending on the position, ortho or meta relative to adjacent hydroxyl groups, result in different products that are metabolized through parallel pathways for entry into the TCA cycle. These degradation pathways differ in the combination of succinate, acetyl-CoA, and pyruvate produced, the reducing equivalents regenerated, and the amount of carbon emitted as $\mathrm{CO}_{2}-$ factors that will ultimately impact the yield of the targeted product. As shown here, the ring-cleavage pathways can be interchanged with one another, and such substitutions have a predictable and substantial impact on product yield. We demonstrate that replacement of the ortho catechol degradation pathway endogenous to Pseudomonas putida KT2440 with an exogenous meta-cleavage pathway from $P$. putida $\mathrm{mt}-2$ increases yields of pyruvate produced from aromatic molecules in engineered strains. Even more dramatically, replacing the endogenous protocatechuate ortho pathway with a meta-cleavage pathway from Sphingobium sp. SYK-6 results in a nearly five-fold increase in pyruvate production. We further demonstrate the aerobic conversion of pyruvate to L-lactate with a yield of $41.1 \pm 2.6 \%$ (wt/wt). Overall, this study illustrates how aromatic degradation pathways can be tuned to optimize the yield of a desired product in biological lignin upgrading.
\end{abstract}

\section{Introduction}

Lignin is a branched, aromatic polymer found in the cell walls of plants that accounts for 15 to $30 \%$ of the dry weight of terrestrial biomass. While it contains more energy and a lower oxygen-to-carbon ratio than either cellulose or hemicellulose (Ragauskas et al., 2014), the structural heterogeneity and recalcitrance have limited its utility in lignocellulosic upgrading strategies, where it is routinely slated for combustion to generate process heat (Chundawat et al., 2011; Ragauskas et al., 2014; Zakzeski et al., 2010).
Recent reports have suggested, however, that incorporation of lignin in upgrading strategies is critical for the economic viability of bio-based fuels and chemicals (Davis et al., 2013; Ragauskas et al., 2014).

When depolymerized in nature by oxidative enzymes secreted by ligninolytic fungi and bacteria, lignin yields a heterogeneous mixture of aromatic monomers that many bacteria use as sources of carbon and energy (Bugg et al., 2011a; 2011b; Martínez et al., 2005). In these organisms, "upper pathways" degrade such mixtures to form conserved
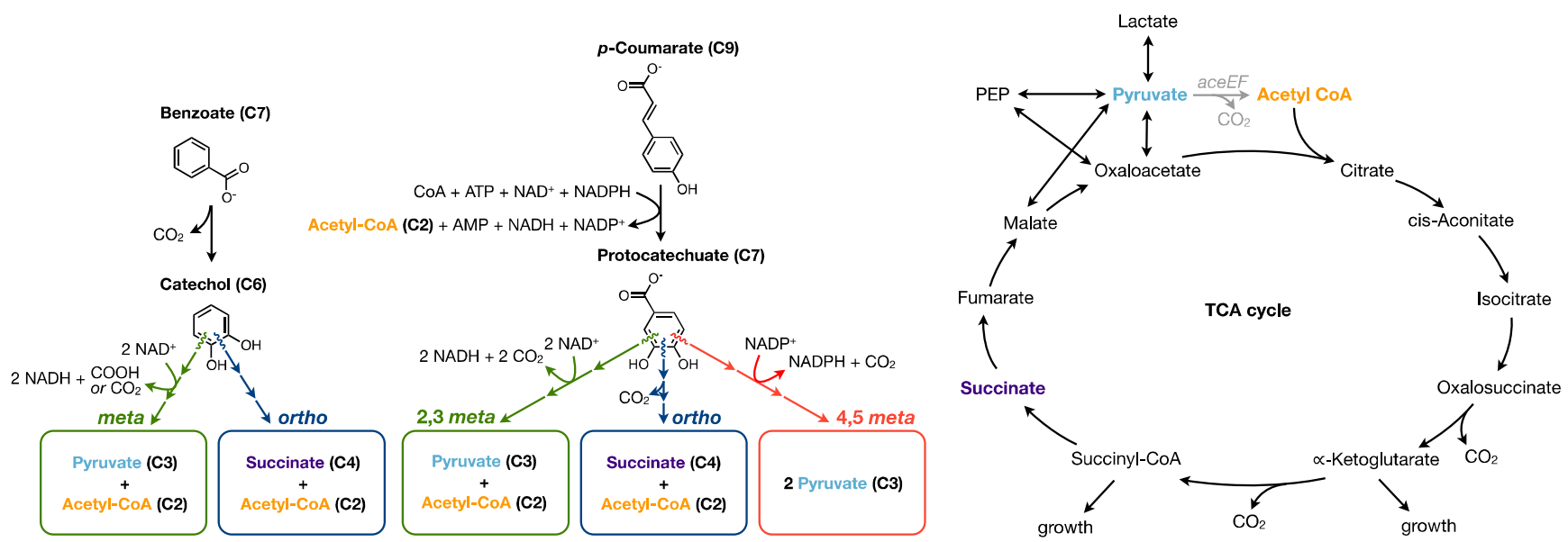

Fig 1. The catechol and protocatechuate ortho and meta degradation pathways. Aromatic molecules such as benzoate and $p$ aerobically through intermediates catechol and protocatechuate. $\mathrm{O}_{2}$-dependent dioxygenase enzymes cleave the aromatic rings of catechol or ortho or meta to the hydroxyl groups. The different products of ring cleavage are degraded through separate pathways arrows) and enter the TCA cycle as different combinations of acetyl-CoA, succinate and pyruvate. Deletion of aceEF pyruvate dehydrogenase complex, prevents the conversion of pyruvate to acetyl-CoA. omitted for simplicity. See the main text for further details. 
metabolic intermediates, acting as a "biological funnel" to reduce the heterogeneity of carbon for catabolism. In aerobic organisms, these central intermediates, which include catechol (1,2-dihydroxybenzene) and protocatechuate $(3,4$-dihydroxybenzoate), are then subjected to $\mathrm{O}_{2}$ dependent ring opening by dioxygenase enzymes that exhibit either ortho (intradiol) or meta (extradiol) cleavage, between or adjacent to the hydroxyl groups, respectively (Fuchs et al., 2011). The cleavage products are degraded through distinct pathways that yield different molecules for entry into central metabolism (Fig. 1). The ortho-cleavage pathways of both catechol and protocatechuate produce succinate and acetyl-CoA (Jiménez et al., 2002). The metacleavage of catechol, as well as the protocatechuate 2,3 meta-cleavage pathway yield pyruvate and acetyl-CoA, while the 4,5 meta-cleavage pathway of protocatechuate ultimately yields two pyruvate molecules (Greated et al., 2002; Harayama and Rekik, 1990; Kamimura et al., 2010; Kamimura and Masai, 2014; Kasai et al., 2009).

Descriptions of aromatic catabolism date back to the 1960 s (Crawford, 1975; Dagley et al., 1960; Dagley and Gibson, 1965; Feist and Hegeman, 1969a; 1969b; Hegeman, 1966; Ornston, 1966; Ornston and Stanier, 1966). Research during the decades since has led to a wealth of knowledge regarding the enzymes that comprise these pathways, the genes that encode them, and the regulatory mechanisms that govern their activity (Fuchs et al., 2011; Harwood and Parales, 1996; Kamimura and Masai, 2014; Pérez-Pantoja et al., 2010). Until recently, the application of these pathways in the synthesis of bio-based products has been limited to just a few examples, such as the production of muconic acid, an intermediate in the catechol ortho degradation pathway, for conversion to adipic acid (Curran et al., 2013; Draths and Frost, 1994; Lin et al., 2014; van Duuren et al., 2012; Weber et al., 2012).

Nature has overcome the heterogeneity of molecules derived from lignin by evolving pathways that funnel carbon into central metabolism through a few common intermediates. Going beyond single intermediates, harnessing this evolutionary innovation to selectively convert a heterogeneous slate of lignin-derived molecules into fuels and chemicals represents a substantial opportunity, as demonstrated by our recent description of medium-chainlength polyhydroxyalkanoate (mcl-PHA) production from alkaline pretreated lignin (Linger et al., 2014). As we move toward utilizing lignin-derived substrates, it is likely that the appropriate deployment of the aromatic catabolic pathways described above will be central to achieving optimal yields of targeted molecules. In order to demonstrate this concept, we have constructed genetically integrated strains that degrade catechol or protocatechuate through ortho or metacleavage pathways using Pseudomonas putida KT2440, a fast growing, genetically amenable, and stress tolerant soil microbe, as the host (Nicolaou et al., 2010; Nikel and de Lorenzo, 2013; Nikel et al., 2014; Poblete-Castro et al., 2013). These strains were evaluated for production of pyruvate and subsequent conversion to L-lactate. Pyruvate is a key intermediate in central metabolism that is fundamental to metabolic engineering strategies for biobased production of amino acids, alcohols including ethanol and isobutanol, terpenoids such as isoprene and farnesene, and lactate (Chandran et al., 2011; Lamsen and Atsumi, 2012; Lee et al., 2004; Park et al., 2007; Zhao et al., 2011; Zhu and Shimizu, 2004). Using our engineered strains, we show that higher yields of pyruvate from aromatic molecules degraded through catechol and protocatechuate can be achieved by utilizing exogenous meta-cleavage pathways rather than the endogenous ortho-cleavage pathways. We then demonstrate the aerobic conversion of pyruvate to L-lactate through the incorporation of a bovine lactate dehydrogenase, ultimately increasing product yield by providing greater competition for pyruvate that might otherwise enter the TCA cycle. Our findings here serve as an example of the benefit that appropriate selection of aromatic degradation pathways can have on biological lignin upgrading.

\section{Materials and methods}

\subsection{Plasmid construction}

Q5 ${ }^{\circledR}$ Hot Start High-Fidelity 2X Master Mix (New England Biolabs) and primers synthesized by Integrated DNA Technologies (IDT) were used in all PCR amplification for plasmid construction. Plasmids were constructed using Gibson Assembly ${ }^{\circledR}$ Master Mix (New England Biolabs) according to the manufacturer's instructions. Plasmids were transformed into competent NEB 5-alpha $\mathrm{F}^{\prime} I^{q} E$. coli (New England Biolabs) according to the manufacturer's instructions. Transformants were selected on LB (Lennox) plates containing $10 \mathrm{~g} / \mathrm{L}$ tryptone, $5 \mathrm{~g} / \mathrm{L}$ yeast extract, $5 \mathrm{~g} / \mathrm{L}$

Table 1.P. putida strains constructed for pyruvate production

\begin{tabular}{|c|c|c|}
\hline Strain & Degradation pathway & Genotype \\
\hline CJ113 & Catechol ortho & $\triangle a c e E F$ Ptac:catBCA \\
\hline CJ112 & Catechol meta & $\triangle a c e E F \Delta c a t A 2 \Delta c a t B C A:: P t a c: x y l E G F J Q K I H^{P . p . m t-2}$ \\
\hline CJ116 & Protocatechuate ortho & $\triangle a c e E F$ Ptac:pcaHG \\
\hline CJ115 & Protocatechuate meta & $\triangle a c e E F \Delta p c a H G:: \mathrm{Ptac}:$ ligABCIUJK $K^{\text {Spp.SYK-6(opt) }}$ \\
\hline
\end{tabular}

Table 2. P. putida strains constructed for L-lactate production

\begin{tabular}{|c|c|c|}
\hline Strain & Degradation pathway & Genotype \\
\hline CJ120 & Catechol ortho & 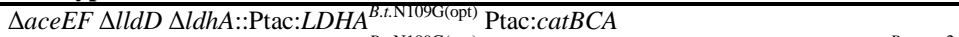 \\
\hline CJ127 & Catechol meta & $\Delta a c e E F \Delta l l d D \Delta l d h A:: \mathrm{Ptac}: L D H A^{\text {B.t.N10yG(opt) }} \Delta c a t A 2 \Delta c a t B C A:: \mathrm{Ptac}: x y l E G F J Q K I H^{P . p . \mathrm{mt}-2}$ \\
\hline CJ124 & Protocatechuate ortho & $\Delta$ aceEF $\Delta l l d D \Delta l d h A:: \mathrm{Ptac}: L D H A^{\text {B.t.N10YG(opt) }}$ Ptac:pcaHG \\
\hline CJ122 & Protocatechuate meta & $\Delta a c e E F \Delta l l d D \Delta l d h A::$ Ptac:LDHA $A^{\text {B.t.N109G(opt) }} \Delta p c a H G::$ Ptac:ligABCIUJK $K^{\text {S.sp. SYK-6 (opt) }}$ \\
\hline
\end{tabular}


$\mathrm{NaCl}$, and $15 \mathrm{~g} / \mathrm{L}$ agar, supplemented with either $10 \mu \mathrm{g} / \mathrm{mL}$ tetracycline or $50 \mu \mathrm{g} / \mathrm{mL}$ kanamycin grown at $37^{\circ} \mathrm{C}$. The sequences of all plasmid inserts were confirmed using Sanger sequencing performed by GENEWIZ, Inc.. Specific plasmid construction details can be found in the supplementary materials and methods.

\subsection{Strain construction}

P. putida KT2440 (ATCC 47054) was used as the basis of strain engineering and gene replacements were made using the antibiotic/sacB system of selection and counter-selection (Marx, 2008). To prepare cells of $P$. putida KT2440 and strains derived from it for transformation by electroporation, LB broth was inoculated to an $\mathrm{OD}_{600}$ of about 0.02 and incubated shaking at $225 \mathrm{rpm}, 30^{\circ} \mathrm{C}$, until an $\mathrm{OD}_{600}$ of $0.5-$ 0.7 was reached. Cells were then centrifuged at $4^{\circ} \mathrm{C}$, washed twice in ice-cold water and once in ice-cold $10 \%$ glycerol before being resuspended in 1/100 of the culture's original volume of $10 \%$ glycerol. Cells were then stored at $-80^{\circ} \mathrm{C}$ or transformed by electroporation immediately. For transformation, $5 \mu \mathrm{L}(200 \mathrm{ng}-2 \mu \mathrm{g})$ of plasmid DNA was added to $50 \mu \mathrm{L}$ of the electrocompetent cells, transferred to a chilled $0.1 \mathrm{~cm}$ electroporation cuvette, and electroporated at $1.6 \mathrm{kV}, 25 \mathrm{uF}, 200 \mathrm{ohms}$. $450 \mu \mathrm{L}$ SOC outgrowth medium (NEB) was added to the cells immediately after electroporation and the resuspended cells were incubated shaking at $225 \mathrm{rpm}, 30^{\circ} \mathrm{C}$, for one hour. The entire transformation was plated on an LB agar plate containing appropriate antibiotics $(30 \mu \mathrm{g} / \mathrm{mL}$ tetracycline for pCM433based plasmids, $50 \mu \mathrm{g} / \mathrm{mL}$ kanamycin for pK18mobsacBbased plasmids) and incubated at $30^{\circ} \mathrm{C}$ overnight. Transformants were restreaked for single colonies on LB agar and incubated at $30^{\circ} \mathrm{C}$ overnight to reduce the possibility of untransformed cells being transferred. For sucrose counter-selection, restreaked transformants were streaked for single colonies on YT $+25 \%$ sucrose plates $(10$ $\mathrm{g} / \mathrm{L}$ yeast extract, $20 \mathrm{~g} / \mathrm{L}$ tryptone, $250 \mathrm{~g} / \mathrm{L}$ sucrose, $18 \mathrm{~g} / \mathrm{L}$ agar), and incubated at $30^{\circ} \mathrm{C}$ overnight. $P$. putida KT2440 containing the $\operatorname{sac} B$ gene can grow, although very slowly, on YT $+25 \%$ sucrose media. Therefore, colonies presumed to have recombined the $s a c B$ gene out of the genome - those colonies that were larger than most - were restreaked on $\mathrm{YT}+25 \%$ sucrose plates and incubated at $30^{\circ} \mathrm{C}$ overnight to reduce the possibility that cells that had not recombined would be carried along with sucrose resistant isolates. Colonies from the second $\mathrm{YT}+25 \%$ sucrose plates were subjected to colony PCR to check for gene replacement at both junctions. These isolates were also plated on LB plates containing appropriate antibiotics to ensure that they had lost antibiotic resistance and, thus, represented pure gene replacements.

\subsection{Culture growth and metabolite analysis}

Shake flask experiments were performed using modified M9 minimal media ( $\mathrm{pH} 7.2$ ) containing $13.56 \mathrm{~g} / \mathrm{L}$ disodium phosphate, $6 \mathrm{~g} / \mathrm{L}$ monopotassium phosphate, $1 \mathrm{~g} / \mathrm{L} \mathrm{NaCl}, 2$ $\mathrm{g} / \mathrm{L} \mathrm{NH}_{4} \mathrm{Cl}, 2 \mathrm{mM} \mathrm{MgSO}{ }_{4}, 100 \mu \mathrm{M} \mathrm{CaCl}_{2}$, and $18 \mu \mathrm{M}$ $\mathrm{FeSO}_{4}$ supplemented with $20 \mathrm{mM}$ Na benzoate (Sigma Aldrich) or $p$-coumaric acid (Sigma Aldrich) neutralized with $\mathrm{NaOH}$. Culture growth was followed by periodic measurement of the optical density at $600 \mathrm{~nm}\left(\mathrm{OD}_{600}\right)$ using a Beckman DU640 spectrophotometer (Beckman Coulter, Brea CA). Concentrations of benzoate, $p$-coumarate, pyruvate, and L-lactate in filtered culture supernatant samples were measured by high performance liquid chromatography (HPLC) on an Agilent1100 series system (Agilent USA, Santa Clara, CA) utilizing a Phenomenex Rezex RFQ-Fast Fruit H+ column (Phenomenex, Torrance, $\mathrm{CA}$ ) and cation $\mathrm{H}+$ guard cartridge (Bio-Rad Laboratories, Hercules, CA) at $85^{\circ} \mathrm{C}$. A mobile phase of $0.1 \mathrm{~N}$ sulfuric acid was used at a flow rate of $1.0 \mathrm{~mL} / \mathrm{min}$ and a diode array detector was utilized for compound detection. Products were identified by comparing the retention times and spectral profiles with pure compounds. Standard deviations were calculated using the following equation:

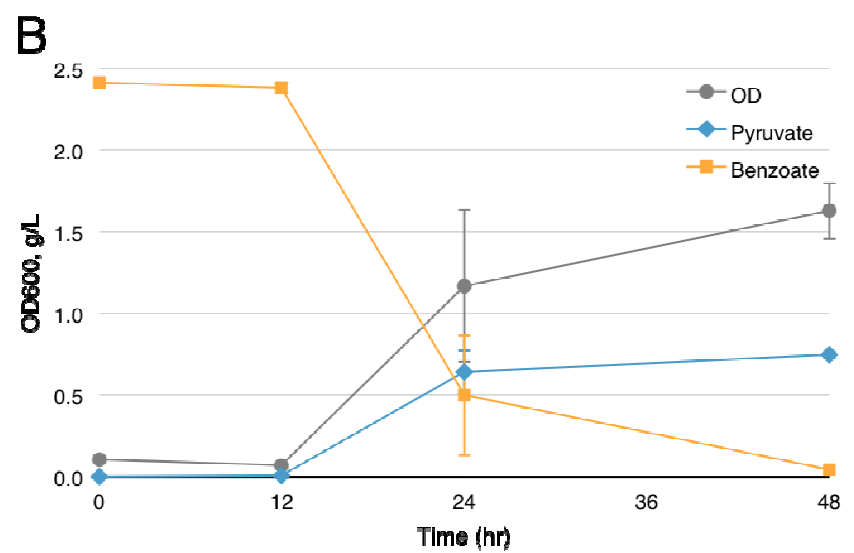

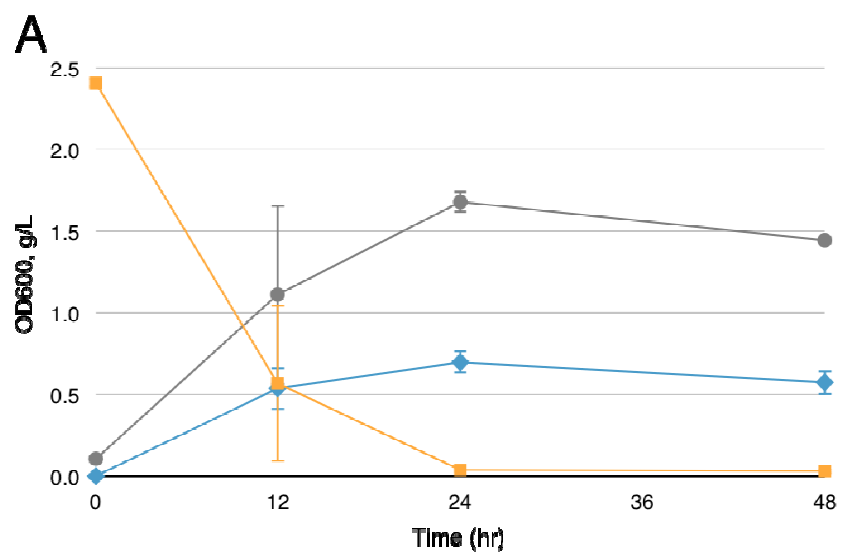

Fig 2. Production of pyruvate from benzoate by engineered $P$. putida strains. Strains were grown in M9 minimal media containing benzoate as the sole carbon and energy source and sampled periodically to evaluate culture growth by OD600 and measure benzoate and pyruvate content by HPLC. Each line | represents the average of three biological replicates. The error bars represent standard deviation of the measurements. (A) The catechol ortho strain, CJ113. (B) The catechol meta strain, CJ112. 


$$
\sqrt{\frac{\sum(x-\bar{x})^{2}}{(n-1)}}
$$

in which $x$ is each value in a sample, $\bar{x}$ is the average of the values, and $n$ is the number of values.

\section{Results}

To demonstrate the influence of aromatic degradation pathway choice on product yield, we chose pyruvate as our target. In order to produce pyruvate in $P$. putida, which does not normally accumulate appreciable amounts of this molecule, we first deleted $a c e E F$, two genes that encode components of the pyruvate dehydrogenase complex that converts pyruvate to acetyl-CoA under aerobic conditions (Tomar et al., 2003). During growth on sugars, this represents the only means of acetyl-CoA production. As expected, we found that our $P$. putida aceEF deletion strain was unable to grow on glucose alone, but that robust growth was achieved with the addition of acetate as a source of acetyl-CoA (data not shown). During growth on most aromatic molecules metabolized through catechol or protocatechuate, acetyl-CoA is produced independent of pyruvate along with either succinate if the native ortho degradation pathways are used or pyruvate if an exogenous meta-cleavage pathway is introduced instead (Fig. 1). In wild-type $P$. putida KT2440, succinate and pyruvate are converted to either oxaloacetate or acetyl-CoA, which are then condensed to form citrate as part of the TCA cycle (del Castillo et al., 2007). Intermediates generated in the TCA cycle are either removed for conversion to molecules required for growth or allowed to complete the cycle to regenerate oxaloacetate. Thus, every cycle requires an acetyl-CoA but may not require succinate or pyruvate if the cycle was completed to regenerate oxaloacetate. Upon deletion of aceEF, however, pyruvate cannot be converted to acetyl-CoA but can still be converted, along with succinate, to oxaloacetate. Based on this, we hypothesized that in carbon-limited cultures grown on benzoate using the

A

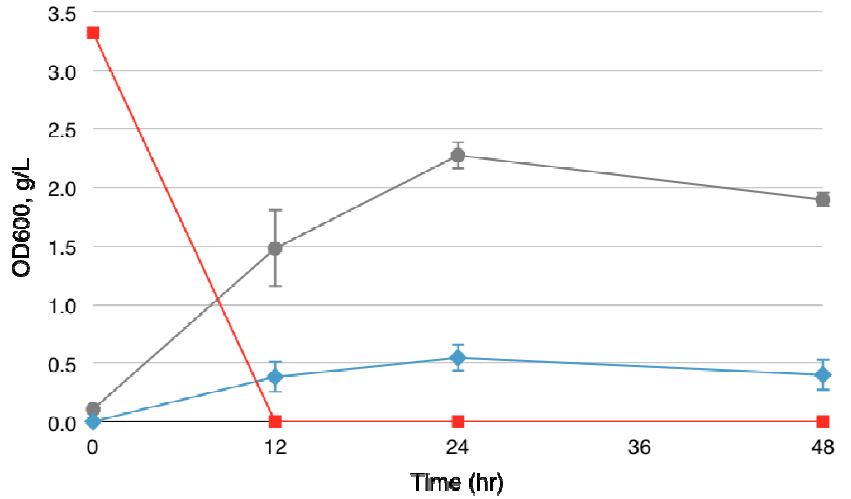

Table 3. Pyruvate yields from engineered strains. Values represent the average and standard deviation of biological triplicates.

\begin{tabular}{llccc}
\hline & Pyruvate & $\begin{array}{c}\text { Maximum } \\
\text { theoretical } \\
\text { pyruvate } \\
\text { \% yield } \\
\text { (wt/wt) }\end{array}$ & $\begin{array}{c}\text { Percent of } \\
\text { maximum } \\
\text { theoretical } \\
\text { pyruvate } \\
\text { \% yield } \\
(\mathbf{w t} / \mathbf{w t})\end{array}$ \\
\hline Strain & $\begin{array}{l}\text { Degradation } \\
\text { pathway }\end{array}$ & $23.9 \pm 3.1$ & 72.1 & $33.1 \pm 0.04$ \\
$\mathbf{C J 1 1 3}$ & Catechol ortho & $31.0 \pm 0.9$ & 72.1 & $43.0 \pm 0.01$ \\
$\mathbf{C J 1 1 2}$ & Catechol meta & 53.6 & $22.6 \pm 0.07$ \\
$\mathbf{C J 1 1 6}$ & Protocatechuate ortho & $12.1 \pm 3.9$ & 5.07 \\
CJ115 & Protocatechuate meta & $59.0 \pm 1.3$ & 107.3 & $55.0 \pm 0.01$
\end{tabular}

native catechol ortho-cleavage pathway and lacking AceEF, growth would occur until acetyl-CoA was diminished, after which any succinate remaining would be converted to pyruvate as the cell attempts futilely to produce more acetylCoA.

To test this hypothesis, we constructed a $P$. putida KT2440derived strain in which we deleted aceEF and integrated the tac promoter uptream of the catBCA operon, which encodes the first three enzymes of the catechol ortho pathway. The tac promoter is expressed constitutively in P. putida (Bagdasarian et al., 1983) and, in the interest of consistency, was used to drive expression of the degradation pathways in all of our engineered strains. We found that growth on benzoate, which is metabolized through catechol, resulted in $23.9 \pm 3.1 \%(\mathrm{wt} / \mathrm{wt})$ of the carbon fed in batch shake-flask experiments to accumulate as pyruvate (Fig. 2). A similar strain in which $a c e E F$ had also been deleted but that lacked the integration of the tac promoter upstream of catBCA performed similarly (Fig. S1, Table S5).

The catechol meta pathway produces one molecule of acetyl-CoA and one pyruvate from each molecule of catechol. Although the two pathways have the same maximum theoretical yield, we hypothesized that deletion of aceEF would result in greater pyruvate accumulation from benzoate if the meta pathway were used since it gives rise to pyruvate directly rather than having to be converted from succinate in direct competition with reactions of the TCA cycle as in the ortho pathway. To examine this, we replaced the catBCA with the operon encoding the meta pathway,

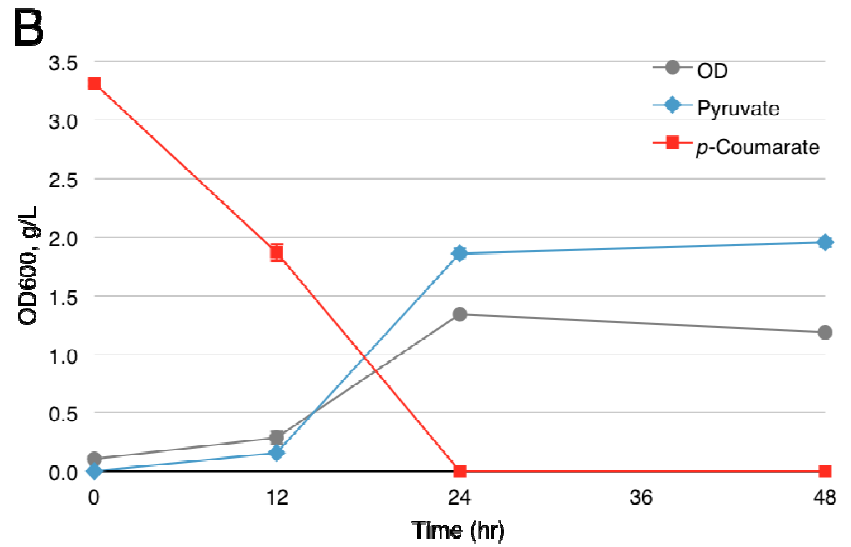

Fig 3. Production of pyruvate from $p$-coumarate by engineered $P$. putida strains. Strains were grown in M9 minimal media containing $p$-coumarate as the sole carbon and energy source and sampled periodically to evaluate culture growth by OD600 and measure $p$-coumarate and pyruvate content by HPLC. Each line represents the average of three biological replicates. The error bars represent standard deviation of the measurements. (A) The protocatechuate ortho strain, CJ116. (B) The protocatechuate 4,5 meta strain, CJ115. 
A

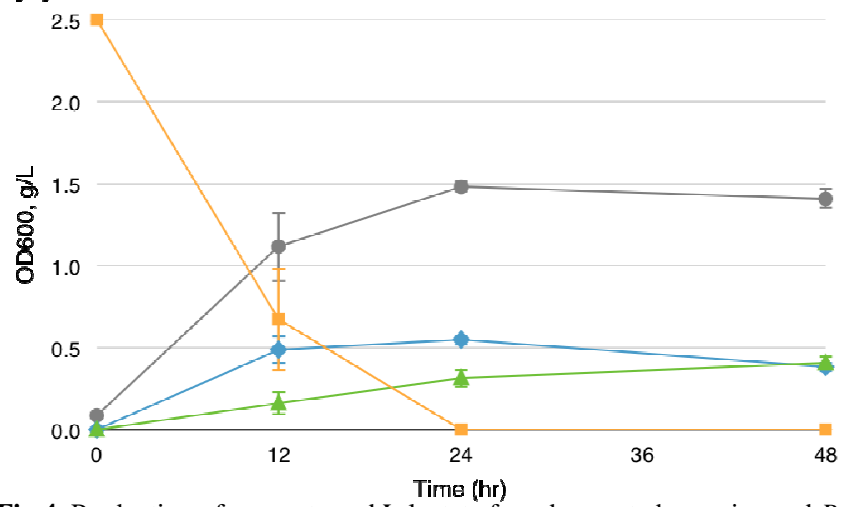

B

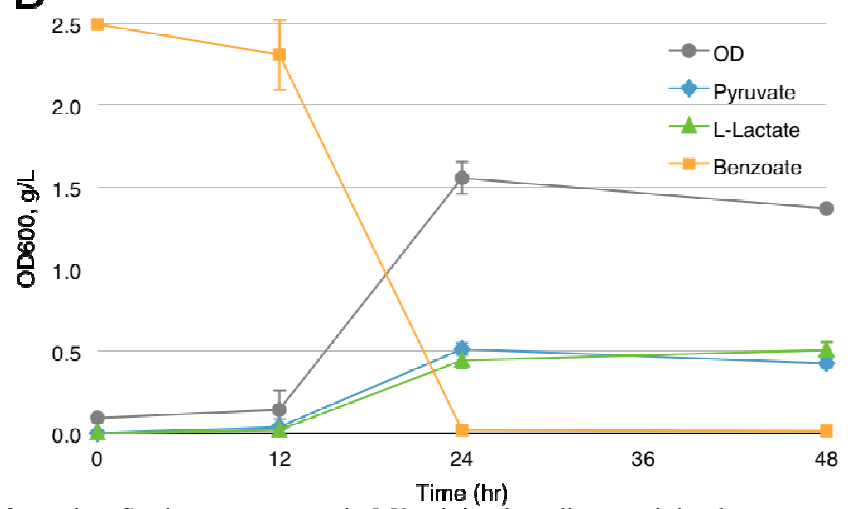

Fig 4. Production of pyruvate and L-lactate from benzoate by engineered $P$. putida strains. Strains were grown in M9 minimal media containing benzoate as the sole carbon and energy source and sampled periodically to evaluate culture growth by OD600 and measure benzoate and pyruvate content by HPLC. Each line represents the average of three biological replicates. The error bars represent standard deviation of the measurements. (A) The catechol ortho strain, CJ120. (B) The catechol meta strain, CJ127.

xylEGFJQKIH, from $P$. putida $\mathrm{mt}-2$, a strain that is essentially identical to KT2440 (Regenhardt et al., 2002) except that it maintains an additional, well studied plasmid, pWW0, that contains this operon (Greated et al., 2002; Harayama and Rekik, 1990). Again, the tac promoter was included to ensure strong transcription of this operon. We also deleted catA2, which is located within the ben operon and encodes a second catechol dioxygenase (van Duuren et al., 2011). We found that this catechol meta degradation strain yielded $31.0 \pm 0.9 \%(\mathrm{wt} / \mathrm{wt})$ pyruvate from benzoate, a small but statistically significant increase from that produced using the catechol ortho pathway in the same growth conditions (Fig. 2).

Next we compared the pyruvate yields achieved using the protocatechuate degradation pathways. P. putida KT2440 natively uses an ortho-cleavage pathway to degrade protocatechuate and, like the catechol ortho pathway, this pathway yields one acetyl-CoA and one succinate from each molecule of protocatechuate. To evaluate production of pyruvate using this pathway, we constructed a strain in which aceEF was deleted and the tac promoter was integrated upstream of the $p c a H G$ operon, which encodes the two subunit protocatec huate dioxygena se and grew the resulting strain on $p$ coumarate. It should be noted that $p$ coumarate and most other aromatic molecules degraded through protocatechuate, including ferulate and caffeate, yield an additional acetyl-CoA prior to being converted to protocatechuate. Because of this, growth of $p$-coumarate using the protocatechuate ortho degradation pathway yields one succinate and two acetyl-CoA for each molecule of $p$-coumarate. This shifts the balance toward acetyl-CoA relative to the catechol ortho pathway discussed above, so we expected less succinate to be converted to pyruvate than we observed with that strain. Indeed, the yield of pyruvate from $p$-coumarate using the protocatechuate ortho degradation pathway was only $12.1 \pm 3.9 \%$ (wt/wt) (Fig. 3). As with the catechol ortho strain, an aceEF deletion strain without the tac promoter integrated upstream of pcaHG exhibited a similar yield (Fig. S1, Table S5).

As discussed above, two protocatechuate meta degradation pathways have been described. The pathway initiated with 2,3 meta-cleavage yields one pyruvate and one acetyl-CoA, similar to the catechol meta-cleavage pathway. As such, we would expect only a modest increase in pyruvate yield using this pathway relative to the protocatechuate ortho pathway as we had demonstrated in comparing the catechol meta and ortho degradation pathways, so this comparison was not

\section{A}

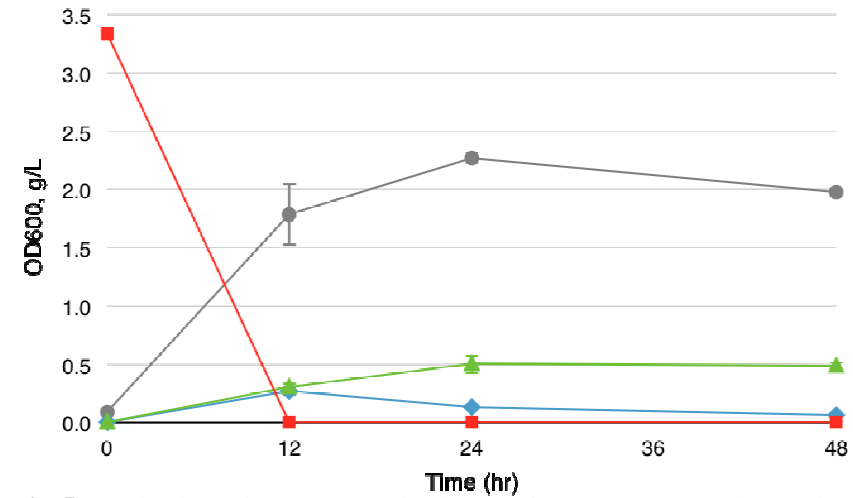

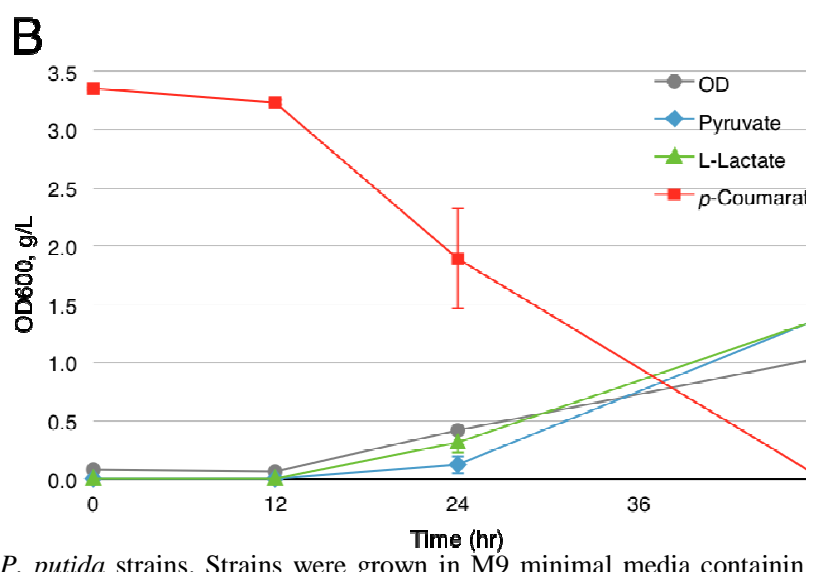

B

ig 5. Production of pyruvate and L-lactate from $p$-coumarate by engineered $P$. putida strains. Strains were grown in M9 minimal media containin coumarate as the sole carbon and energy source and sampled periodically to evaluate culture growth by OD600 and measure $p$-coumarate and pyru content by HPLC. Each line represents the average of three biological replicates. The error bars represent standard deviation of the measurements. (A) protocatechuate ortho strain, CJ124. (B) The protocatechuate 4,5 meta strain, CJ122. 


\begin{tabular}{lllll}
\hline & & & & \\
\end{tabular}

pursued. The protocatechuate 4,5 meta-cleavage pathway yields two molecules of pyruvate and one $\mathrm{CO}_{2}$ for every molecule of protocatechuate, and $p$-coumarate would produce an additional acetyl-CoA. This shifts the balance between pyruvate and acetyl-CoA strongly in direction of pyruvate, so we expected that upon deletion of aceEF we would see a much greater accumulation of pyruvate. To construct a $P$. putida strain that uses this protocatechuate meta pathway, we replaced $p c a H G$ with a synthetic operon of codon-optimized genes encoding this pathway from Sphingobium sp. strain SYK-6, ligABCIUJK (Hara et al., 2003; Kamimura and Masai, 2014; Noda et al., 1990), again driven by the tac promoter. When aceEF was also deleted and the resulting strain was grown in minimal media containing $p$-coumarate, $59.0 \pm 1.3 \%$ (wt/wt) of the carbon accumulated as pyruvate, a substantial increase from the $12.1 \pm 3.9 \%(\mathrm{wt} / \mathrm{wt})$ achieved with the native ortho pathway in the same growth conditions (Fig. 3).

We next sought to apply our findings above to production of lactate. The conversion of pyruvate to $\mathrm{D}(-)$ or $\mathrm{L}(+)$ lactate by lactate dehydrogenase enzymes is evolutionarily conserved, from bacteria to vertebrates, and occurs in order to regenerate NAD+ required for sugar metabolism (Holbrook et al., 1975). Despite being categorized as an aerobe, the $P$. putida KT2440 genome contains an ldhA gene (PP_1649) encoding a D-lactate dehydrogenase very similar to the enzyme that produces D-lactate in E. coli during fermentation (Jiang et al., 2001; Tarmy and Kaplan, 1968). We first constructed a strain that overexpresses this endogenous $l d h A$ gene by integrating the tac promoter upstream of it. This strain failed to produce D-lactate except under microaerobic conditions, during which a very small amount of D-lactate was produced after several days (data not shown). Reasoning that the P. putida LdhA likely only functions enhance survival under intermittent anaerobic conditions (Eschbach et al., 2004) and may not be a robust enzyme, we next tried to produce L-lactate by replacing the endogenous D-lactate $l d h A$ with codon optimized, L-lactate dehydrogenase genes from two lactic acid bacteria, the $l d h$ gene from Lactobacillus casei and the ldhl gene Lactobacillus plantarum (Branduardi et al., 2006; Taguchi and Ohta, 1991). P. putida also contains another lactate dehydrogenase encoded by $l l d D$ (PP_4736) that allows it to convert L-lactate to pyruvate (Escapa et al., 2012), so this gene was deleted from these strains to prevent catabolism of any L-lactate produced. Despite the integration of these robust lactate dehydrogenases and deletion of $l l d D$, the resulting strains failed to produce appreciable amounts of Llactate, even when growing on glucose, which is expected to balance redox (data not shown). The lactate dehydrogenase is highly regulated at transcriptional, translational, and posttranslational levels in response to many environmental cues including, $\mathrm{pH}$, oxygen availability and presence of metabolites such as fructose bis-phosphate (Garvie, 1980; Holbrook et al., 1975). We hypothesized that an evolutionary distant lactate dehydrogenase, such as the Llactate dehydrogenase from Bos taurus that has been utilized successfully for L-lactate production in yeast (Branduardi et al., 2006; Porro et al., 1995), may be less likely to be regulated by the endogenous mechanisms that govern lactate dehydrogenase activity in $P$. putida. The optimal $\mathrm{pH}$ for conversion of pyruvate to L-lactate by the bovine lactate dehydrogenase has been reported to be 7.0, with $>80 \%$ activity from pH 6.0 to 8.0 (Kolb et al., 1970), making it well suited for cultures grown in M9 medium, which is buffered to $\mathrm{pH}$ 7.2. We had synthesized a gene encoding the $B$. taurus lactate dehydrogenase, LDHA, that we codon optimized for expression in P. putida and altered to incorporate an N109G amino acid substitution shown to increase activity of the homologous enzyme in L. plantarum (Branduardi et al., 2006). Upon integration of this gene, $L D H A^{\text {B.t.N109G(opt) }}$, into the $l l d D$ aceEF deletion strain, the resulting strain converted about $50 \%$ (wt/wt) of the pyruvate accumulated to L-lactate when grown aerobically on benzoate or glucose (data not shown). As mentioned before, glucose metabolism regenerates NADH used by the lactate dehydrogenases but cultures grown on glucose and benzoate exhibited similar levels of pyruvate conversion to L-lactate (data not shown), suggesting that NADH availability did not limit lactate dehydrogenase activity in the conditions examined.

We next integrated this $L D H A^{\text {B.t.N109G(opt) }}$ and deleted the lldD gene from our catechol and protocatechuate degradation pathway comparison strains and compared them in shake-flask experiments as before. In all cases, about half of the pyruvate produced was converted to L-lactate, with the exception of the protocatechuate ortho degradation pathway strain, which converted $87 \%$ (wt/wt) of pyruvate to L-lactate (Fig. 4 and 5, Table 4). We expected that the combined amount of pyruvate and L-lactate produced would 
be roughly equivalent to the amount of pyruvate produced in the equivalent strains that lacked the lactate dehydrogenase, but were surprised to find that they were $20-40 \%$ higher (Table 4). Notably, the protocatechuate meta degradation pathway strain achieved an $82.5 \pm 3.5 \%$ (wt/wt) yield of Llactate and pyruvate combined, a $40 \%$ increase from the $59.0 \pm 1.3 \%(w t / w t)$ yield achieved with the analogous pyruvate producing strain.

\section{Discussion and Conclusions}

Biological lignin upgrading strategies will benefit from the evolutionary ingenuity of the microbes they employ. Not only have microbes developed a means of funneling a heterogenous mix of aromatic molecules to form a few central intermediates, they have also developed a diverse set of pathways through which these intermediates can enter central metabolism (Fuchs et al., 2011; Phale et al., 2007; Williams and Sayers, 1994). In this study, we have demonstrated the impact that choosing the appropriate aromatic cleavage pathway can have on product yield. Degradation of catechol and protocatechuate using exogenous meta-cleavage pathways ultimately resulted in greater yields of pyruvate and L-lactate than the native ortho-cleavage pathways. Strains expressing these exogenous pathways also exhibited a notable lag in growth and substrate utilization that we believe might be attributed to the complex regulation of the "upper pathways", which are sometimes induced by downstream degradation intermediates that might not be present in the heterologously expressed degradation pathways (Clark et al., 2004; Cosper et al., 2000). In comparing the catechol ortho and meta pathway, we saw a slight but significant increase in production of both pyruvate and L-lactate from benzoate in strains using the meta pathway (Fig 2., Table 3). While the ortho and meta pathways have the same maximum theoretical yield of pyruvate and L-lactate, we believe the advantage observed in using the meta pathway can be attributed to the fact that this pathway yields pyruvate directly rather than having to be converted from succinate and entering into direct competition with reactions of the TCA cycle. We observed a greater benefit from replacing the endogenous protocatechuate ortho-cleavage pathway with the 4,5 meta pathway, which increased the pyruvate yield nearly five fold (Fig 3., Table 3 ). We believe this can be attributed to a maximum theoretical yield that is twice that of the ortho pathway and the general principal that, in carbon limited cultures lacking the pyruvate dehydrogenase complex $(\triangle a c e E F)$ such as those in this study, the amount of acetyl-CoA generated by aromatic degradation limits the amount of oxaloacetate (or other molecules that can readily be converted to it, including pyruvate and succinate) that will be used to replenish TCA cycle intermediates needed for growth. Degradation of $p$-coumarate using the 4,5 meta pathway gives rise to two pyruvate per acetyl-CoA, resulting in a much greater surplus of pyruvate once acetylCoA has been depleted relative to the protocatechuate ortho-cleavage pathway, which yields one succinate and two acetyl-CoA from each molecule of $p$-coumarate.

We also demonstrated the conversion of pyruvate accumulated in these strains to L-lactate. Lactate dehydrogenases are highly regulated enzymes that are generally reserved for anaerobic growth in nature (Garvie, 1980; Holbrook et al., 1975). Although we were only able to achieve about $50 \%$ (wt/wt) conversion of the pyruvate produced to L-lactate (Table 4), this is not an insignificant achievement given that L-lactate production we demonstrate here occurred in aerobic conditions. Interestingly, we saw a much higher conversion of pyruvate to L-lactate using the protocatechuate ortho pathway relative to the other pathways (Fig. 5A, Table 4). This is difficult to explain given what is known about this pathway and about the regulation of lactate dehydrogenase activity, but it could be that an intermediate molecule in this pathway either directly or indirectly affects activity of this enzyme. The fact that all of the other pathways converted a very similar fraction of pyruvate to L-lactate despite have very different yields of reducing equivalents (Figure 1) suggests that lactate dehydrogenase activity was not affected by redox balance in these experiments. While employing an evolutionarily distant lactate dehydrogenase seems to have been a fruitful strategy for aerobic production of L-lacate in $P$. putida, optimizing culture conditions and/or protein engineering would be useful strategies to increase the activity of this enzyme and will be pursued in future studies.

The conversion of pyruvate to L-lactate also had an interesting effect on the combined yield of pyruvate and Llactate - In the case of all four degradation pathways, the combined yield of pyruvate and L-lactate was higher when pyruvate was converted to L-lactate than when pyruvate was the end product, with the protocatechuate 4,5 meta-cleavage pathways exhibiting a combined yield of $82.5 \pm 3.5 \%$ (wt/wt), which represents $75 \%$ of the theoretical maximum (Table 4). We speculate that conversion of pyruvate to Llactate provides competition that ultimately reduces the flux of pyruvate to oxaloacetate and hypothesize that, if the lactate dehydrogenase were able to convert more pyruvate to L-lactate, the increased competition would result in an even greater combined yield.

In this study, we demonstrated how degradation pathway choice can affect the yield of pyruvate and L-lactate on the basis of how carbon enters the TCA cycle. This concept, however, could be applied to other products. If one were targeting succinate, for instance, the ortho-cleavage pathways would be an obvious choice since these pathways produce it directly (Figure 1). If the product of interest requires reducing equivalents in the form of NADPH, one might find benefit from employing the protocatechuate 4,5 meta pathway, which regenerates it. If additional NADH would be of benefit, one might considering using the protocatechuate 2,3 meta or the catechol meta pathway, both of which regenerate two NADH for each molecule of substrate. In fact, depending on which branch of this 
pathway is used, the catechol meta-cleavage pathway can produce formate that could be further oxidized to $\mathrm{CO}_{2}$ by a formate dehydrogenase to regenerate another NADH (Berríos-Rivera, 2002). If carbon yield takes precedence above all else, one would be advised to avoid the protocatechuate 2,3 meta and catechol meta pathways, which generate more $\mathrm{CO}_{2}$ and, thus, less useful carbon than the other pathways.

The utilization of lignin for production of bio-based chemicals and fuels represents a substantial challenge as well as an enormous opportunity. Incorporating lignin in biomass upgrading strategies could ultimately enable the production of fuels and commodity chemicals that might otherwise be economically intractable (Davis et al., 2013; Ragauskas et al., 2014). The concept of biological funneling (Linger et al., 2014) as a means to valorize lignin will require significant advances to be made in all steps of a potential process, including strategies for metabolic engineering of aromatic-catabolizing organisms. The current study highlights the importance of judicious selection of aromatic cleavage pathways for optimal carbon efficiency and redox balance.

\section{Acknowledgements}

The authors are grateful to US Department of Energy BioEnergy Technologies Office for funding this work. We thank Mary Ann Franden for her assistance with HPLC, Jeffrey Linger for critical reading of the manuscript, and Michael Guarnieri and Philip Pienkos for their valuable input and feedback.

\section{References}

Bagdasarian, M.M., Amann, E., Lurz, R., Rückert, B., Bagdasarian, M., 1983. Activity of the hybrid trp-lac (tac) promoter of Escherichia coli in Pseudomonas putida. Construction of broad-host-range, controlled-expression vectors. Gene 26, 273-282.

Berríos-Rivera, S., 2002. Metabolic Engineering of Escherichia coli: Increase of NADH Availability by Overexpressing an NAD+-Dependent Formate Dehydrogenase. Metabolic Engineering 4, 217-229.

Branduardi, P., Sauer, M., De Gioia, L., Zampella, G., Valli, M., Mattanovich, D., Porro, D., 2006. Lactate production yield from engineered yeasts is dependent from the host background, the lactate dehydrogenase source and the lactate export. Microb. Cell Fact. 5:4.

Bugg, T.D.H., Ahmad, M., Hardiman, E.M., Rahmanpour, R., 2011a. Pathways for degradation of lignin in bacteria and fungi. Nat Prod Rep 28, 1883-1896.

Bugg, T.D.H., Ahmad, M., Hardiman, E.M., Singh, R., 2011b. The emerging role for bacteria in lignin degradation and bio-product formation. Curr. Opin. Biotechnol.

Chandran, S.S., Kealey, J.T., Reeves, C.D., 2011. Microbial production of isoprenoids. Process Biochemistry 46, 1703-1710.

Chundawat, S.P.S., Beckham, G.T., Himmel, M.E., Dale, B.E., 2011. Deconstruction of Lignocellulosic Biomass to Fuels and Chemicals. Annu. Rev. Chem. Biomol. Eng. 2, 121-145.

Clark, T.J., Phillips, R.S., Bundy, B.M., Momany, C., Neidle, E.L., 2004. Benzoate Decreases the Binding of cis,cis-Muconate to the BenM Regulator despite the Synergistic Effect of Both Compounds on Transcriptional Activation. Journal of Bacteriology 186, 1200-1204.

Cosper, N.J., Collier, L.S., Clark, T.J., Scott, R.A., Neidle, E.L., 2000. Mutations in cat $\mathrm{B}$, the gene encoding muconate cycloisomerase, activate transcription of the distal ben genes and contribute to a complex regulatory circuit in Acinetobacter sp. strain ADP1. Journal of Bacteriology 182, 7044-7052.

Crawford, R.L., 1975. Novel pathway for degradation of protocatechuic acid in Bacillus species. Journal of Bacteriology 121, 531-536.

Curran, K.A., Leavitt, J.M., Karim, A.S., Alper, H.S., 2013. Metabolic engineering of muconic acid production in Saccharomyces cerevisiae. Metabolic Engineering $15,55-66$.
Dagley, S., Evans, W.C., Ribbons, D.W., 1960. New pathways in the oxidative metabolism of aromatic compounds by microorganisms. Nature 188, 560-566.

Dagley, S., Gibson, D.T., 1965. The Bacterial Degradation of Catechol. Biochem. J. $95,466-474$.

Davis, R., Tao, L., Tan, E.C.D., Biddy, M.J., Beckham, G.T., Scarlata, C., Jacobson, J., Cafferty, K., Ross, J., Lukas, J., Knorr, D., Schoen, P., 2013. Process Design and Economics for the Conversion of Lignocellulosic Biomass to Hydrocarbons: Dilute-Acid and Enzymatic Deconstruction of Biomass to Sugars and Biological Conversion of Sugars to Hydrocarbons, NREL Technical Report. NREL.

del Castillo, T., Ramos, J.L., Rodriguez-Herva, J.J., Fuhrer, T., Sauer, U., Duque, E., 2007. Convergent Peripheral Pathways Catalyze Initial Glucose Catabolism in Pseudomonas putida: Genomic and Flux Analysis. Journal of Bacteriology $189,5142-5152$

Draths, K.M., Frost, J.W., 1994. Environmentally compatible synthesis of adipic acid from D-glucose. J. Am. Chem. Soc. 116, 399-400.

Escapa, I.F., Garcia, J.L., Bühler, B., Blank, L.M., Prieto, M.A., 2012. The polyhydroxyalkanoate metabolism controls carbon and energy spillage in Pseudomonas putida. Environmental Microbiology 14, 1049-1063.

Eschbach, M., Schreiber, K., Trunk, K., Buer, J., Jahn, D., Schobert, M., 2004. Longterm anaerobic survival of the opportunistic pathogen Pseudomonas aeruginosa via pyruvate fermentation. Journal of Bacteriology 186, 45964604

Feist, C.F., Hegeman, G.D., 1969a. Phenol and benzoate metabolism by Pseudomonas putida: regulation of tangential pathways. Journal of Bacteriology 100, 869877.

Feist, C.F., Hegeman, G.D., 1969b. Regulation of the meta cleavage pathway for benzoate oxidation by Pseudomonas putida. Journal of Bacteriology 100, 1121-1123.

Fuchs, G., Boll, M., Heider, J., 2011. Microbial degradation of aromatic compounds from one strategy to four. Nature Reviews Microbiology 9, 803-816.

Garvie, E.I., 1980. Bacterial lactate dehydrogenases. Microbiol. Rev. 44, 106-139.

Greated, A., Lambertsen, L., Williams, P.A., Thomas, C.M., 2002. Complete sequence of the IncP-9 TOL plasmid pWW0 from Pseudomonas putida. Environmental Microbiology 4, 856-871.

Hara, H., Masai, E., Miyauchi, K., Katayama, Y., Fukuda, M., 2003. Characterization of the 4-carboxy-4-hydroxy-2-oxoadipate aldolase gene and operon structure of the protocatechuate 4, 5-cleavage pathway genes in Sphingomonas paucimobilis SYK-6. Journal of Bacteriology 185, 41-50.

Harayama, S., Rekik, M., 1990. The meta cleavage operon of TOL degradative plasmid pWW0 comprises 13 genes. Mol. Gen. Genet. 221, 113-120.

Harwood, C.S., Parales, R.E., 1996. The beta-ketoadipate pathway and the biology of self-identity. Annu. Rev. Microbiol. 50, 553-590.

Hegeman, G.D., 1966. Synthesis of the enzymes of the mandelate pathway by Pseudomonas putida. I. Synthesis of enzymes by the wild type. Journal of Bacteriology 91, 1140-1154.

Holbrook, J.J., Liljas, A., Steindel, S.J., Rossmann, M.G., 1975. Lactate Dehydrogenase, in: The Enzymes. Elsevier, pp. 191-292.

Jiang, G.R., Nikolova, S., Clark, D.P., 2001. Regulation of the ldhA gene, encoding the fermentative lactate dehydrogenase of Escherichia coli. Microbiology (Reading, Engl.) 147, 2437-2446.

Jiménez, J.I., Miñambres, B., García, J.L., Díaz, E., 2002. Genomic analysis of the aromatic catabolic pathways from Pseudomonas putida KT2440. Environmental Microbiology 4, 824-841.

Kamimura, N., Aoyama, T., Yoshida, R., Takahashi, K., Kasai, D., Abe, T., Mase, K., Katayama, Y., Fukuda, M., Masai, E., 2010. Characterization of the Protocatechuate 4,5-Cleavage Pathway Operon in Comamonas sp. Strain E6 and Discovery of a Novel Pathway Gene. Applied and Environmental Microbiology 76, 8093-8101.

Kamimura, N., Masai, E., 2014. The Protocatechuate 4, 5-Cleavage Pathway: Overview and New Findings, in: Nojiri, H., Tsuda, M., Fukuda, M., Kamagata, Y. (Eds.), Biodegradative Bacteria: How Bacteria Degrade, Survive, Adapt, and Evolve. Biodegradative Bacteria, pp. 207-226.

Kasai, D., Fujinami, T., Abe, T., Mase, K., Katayama, Y., Fukuda, M., Masai, E., 2009. Uncovering the protocatechuate 2,3-cleavage pathway genes. Journal of Bacteriology 191, 6758-6768.

Kolb, E., Fleisher, G.A., Larner, J., 1970. Isolation and characterization of bovine lactate dehydrogenase X. Biochemistry 9, 4372-4380.

Lamsen, E.N., Atsumi, S., 2012. Recent progress in synthetic biology for microbial production of C3-C10 alcohols. Front Microbiol 3.

Lee, M., Smith, G.M., Eiteman, M.A., Altman, E., 2004. Aerobic production of alanine by Escherichia coli aceF ldhA mutants expressing the Bacillus sphaericus alaD gene. Applied Microbiology and Biotechnology 65, 56-60.

Lin, Y., Sun, X., Yuan, Q., Yan, Y., 2014. Extending shikimate pathway for the production of muconic acid and its precursor salicylic acid in Escherichia coli. Metabolic Engineering 23, 62-69.

Linger, J.G., Vardon, D.R., Guarnieri, M.T., Karp, E.M., Hunsinger, G.B., Franden, M.A., Johnson, C.W., Chupka, G., Strathmann, T.J., Pienkos, P.T., Beckham, G.T., 2014. Lignin valorization through integrated biological funneling and chemical catalysis. Proceedings of the National Academy of Sciences 111, 12013-12018.

Martínez, A.T., Speranza, M., Ruiz-Dueñas, F.J., Ferreira, P., Camarero, S., Guillén, F., Martínez, M.J., Gutiérrez, A., del Río, J.C., 2005. Biodegradation of lignocellulosics: microbial, chemical, and enzymatic aspects of the fungal 
attack of lignin. Int. Microbiol. 8, 195-204.

Marx, C.J., 2008. Development of a broad-host-range sacB-based vector for unmarked allelic exchange. BMC Res Notes 1:1.

Nicolaou, S.A., Gaida, S.M., Papoutsakis, E.T., 2010. A comparative view of metabolite and substrate stress and tolerance in microbial bioprocessing From biofuels and chemicals, to biocatalysis and bioremediation. Metabolic Engineering 12, 307-331.

Nikel, P.I., de Lorenzo, V., 2013. Engineering an anaerobic metabolic regime in Pseudomonas putida KT2440 for the anoxic biodegradation of 1,3dichloroprop-1-ene. Metabolic Engineering 15, 98-112.

Nikel, P.I., Martínez-García, E., de Lorenzo, V., 2014. Biotechnological domestication of pseudomonads using synthetic biology. Nature Reviews Microbiology 12, 368-379.

Noda, Y., Nishikawa, S., Shiozuka, K., Kadokura, H., Nakajima, H., Yoda, K., Katayama, Y., Morohoshi, N., Haraguchi, T., Yamasaki, M., 1990. Molecular cloning of the protocatechuate 4, 5-dioxygenase genes of Pseudomonas paucimobilis. Journal of Bacteriology 172, 2704-2709.

Ornston, L.N., 1966. The conversion of catechol and protocatechuate to betaketoadipate by Pseudomonas putida. IV. Regulation. J. Biol. Chem. 241, 3800-3810.

Ornston, L.N., Stanier, R.Y., 1966. The conversion of catechol and protocatechuate to beta-ketoadipate by Pseudomonas putida. J. Biol. Chem. 241, 3776-3786.

Park, J.H., Lee, K.H., Kim, T.Y., Lee, S.Y., 2007. Metabolic engineering of Escherichia coli for the production of L-valine based on transcriptome analysis and in silico gene knockout simulation. Proc. Natl. Acad. Sci. U.S.A. 104, 7797-7802.

Pérez-Pantoja, D., González, B., Pieper, D.H., 2010. Aerobic Degradation of Aromatic Hydrocarbons, in: Handbook of Hydrocarbon and Lipid Microbiology. Springer Berlin Heidelberg, Berlin, Heidelberg, pp. 799-837.

Phale, P.S., Basu, A., Majhi, P.D., Deveryshetty, J., Vamsee-Krishna, C., Shrivastava, R., 2007. Metabolic diversity in bacterial degradation of aromatic compounds. OMICS 11, 252-279.

Poblete-Castro, I., Binger, D., Rodrigues, A., Becker, J., Martins dos Santos, V.A.P., Wittmann, C., 2013. In-silico-driven metabolic engineering of Pseudomonas putida for enhanced production of poly-hydroxyalkanoates. Metabolic Engineering 15, 113-123.

Porro, D., Brambilla, L., Ranzi, B.M., Martegani, E., Alberghina, L., 1995. Development of metabolically engineered Saccharomyces cerevisiae cells for the production of lactic acid. Biotechnol. Prog. 11, 294-298.

Ragauskas, A.J., Beckham, G.T., Biddy, M.J., Chandra, R., Chen, F., Davis, M.F., Davison, B.H., Dixon, R.A., Gilna, P., Keller, M., Langan, P., Naskar, A.K., Saddler, J.N., Tschaplinski, T.J., Tuskan, G.A., Wyman, C.E., 2014. Lignin valorization: improving lignin processing in the biorefinery. Science 344 , 1246843.

Regenhardt, D., Heuer, H., Heim, S., Fernandez, D.U., Strompl, C., Moore, E., Timmis, K.N., 2002. Pedigree and taxonomic credentials of Pseudomonas putida strain KT2440. Environmental Microbiology 4, 912-915.

Taguchi, H., Ohta, T., 1991. D-lactate dehydrogenase is a member of the D-isomerspecific 2-hydroxyacid dehydrogenase family. Cloning, sequencing, and expression in Escherichia coli of the D-lactate dehydrogenase gene of Lactobacillus plantarum. J. Biol. Chem. 266, 12588-12594.

Tarmy, E.M., Kaplan, N.O., 1968. Chemical characterization of D-lactate dehydrogenase from Escherichia coli B. J. Biol. Chem. 243, 2579-2586.

Tomar, A., Eiteman, M.A., Altman, E., 2003. The effect of acetate pathway mutations on the production of pyruvate in Escherichia coli. Applied Microbiology and Biotechnology 62, 76-82.

van Duuren, J.B.J.H., Wijte, D., Karge, B., Santos, dos, V.A.P.M., Yang, Y., Mars, A.E., Eggink, G., 2012. pH-stat fed-batch process to enhance the production of cis, cis-muconate from benzoate by Pseudomonas putida KT2440-JD1. Biotechnol. Prog. 28, 85-92. doi:10.1002/btpr.709

van Duuren, J.B.J.H., Wijte, D., Leprince, A., Karge, B., Puchałka, J., Wery, J., Santos, dos, V.A.P.M., Eggink, G., Mars, A.E., 2011. Generation of a catR deficient mutant of $P$. putida KT2440 that produces cis, cis-muconate from benzoate at high rate and yield. Journal of Biotechnology 156, 163-172.

Weber, C., Brückner, C., Weinreb, S., Lehr, C., Essl, C., Boles, E., 2012. Biosynthesis of cis,cis-muconic acid and its aromatic precursors, catechol and protocatechuic acid, from renewable feedstocks by Saccharomyces cerevisiae. Applied and Environmental Microbiology 78, 8421-8430.

Williams, P.A., Sayers, J.R., 1994. The evolution of pathways for aromatic hydrocarbon oxidation in Pseudomonas. Biodegradation 5, 195-217.

Zakzeski, J., Bruijnincx, P.C.A., Jongerius, A.L., Weckhuysen, B.M., 2010. The Catalytic Valorization of Lignin for the Production of Renewable Chemicals. Chem. Rev. 110, 3552-3599.

Zhao, Y., Yang, J., Qin, B., Li, Y., Sun, Y., Su, S., Xian, M., 2011. Biosynthesis of isoprene in Escherichia coli via methylerythritol phosphate (MEP) pathway. Applied Microbiology and Biotechnology 90, 1915-1922.

Zhu, J., Shimizu, K., 2004. The effect of $p f l$ gene knockout on the metabolism for optically pure D-lactate production by Escherichia coli. Applied Microbiology and Biotechnology 64, 367-375. 


\title{
Aromatic catabolic pathway selection for optimal production of pyruvate and lactate from lignin
}

\author{
Christopher W. Johnson and Gregg T. Beckham* \\ National Bioenergy Center, National Renewable Energy Laboratory, Golden CO 80401 \\ *Corresponding AuthorInformation: gregg.beckham@nrel.gov; +01-303-384-7806
}

\section{SUPPLEMENTAL INFORMATION}

\section{MATERIALS AND METHODS}

Plasmid construction details

Plasmids used for gene replacements and details on their construction are included in Table S1. Table S2 contains the sequences of primers used in construction of these plasmids.

Gene replacement confirmation

Colony PCR using Q5 ${ }^{\circledR}$ Hot Start High-Fidelity 2X Master Mix (New England Biolabs) and primers synthesized by Integrated DNA Technologies was used to confirm gene replacement by amplifying either the entire locus or the 5' and 3' junctions, depending on the size of the replacement. The sequences of primers used for confirmation by colony PCR and the size of the PCR products that indicated gene replacement are included in Table S3.

Table S1. Plasmids used for gene replacements in this study

\begin{tabular}{|c|c|c|}
\hline Plasmid & Purpose & Construction details \\
\hline pCJ004 & To delete catA2 & $\begin{array}{l}\text { The 5' homology region was amplified from P. putida KT2440 genomic DNA with primers oCJ038 (Fwd) and oCJ039 } \\
\text { (Rev) and the 3' homology region was amplified using primers oCJ040 (Fwd) and oCJ041 (Rev). These fragments were } \\
\text { then assembled into pCM433 digested with AatII and SacI. }\end{array}$ \\
\hline pCJ006 & $\begin{array}{l}\text { To integrate the tac } \\
\text { promoter upstream of } \\
\text { catBCA }\end{array}$ & $\begin{array}{l}\text { The 5' homology region was amplified from P. putida KT2440 genomic DNA with primers oCJ042 (Fwd), and oCJ043 } \\
\text { (Rev), which incorporated the tac promoter. The 3' homology region was amplified with oCJ049 (Fwd) and oCJ050 (Rev). } \\
\text { These fragments were then assembled into pCM433 digested with AatII and SacI. }\end{array}$ \\
\hline pCJ007 & $\begin{array}{l}\text { To integrate the tac } \\
\text { promoter upstream of } \\
\text { ldhA and used as a } \\
\text { template in construction } \\
\text { of pCJ015 }\end{array}$ & $\begin{array}{l}\text { The 5' homology region was amplified from P. putida KT2440 genomic DNA with primers oCJ077 (Fwd) and oCJ078 } \\
\text { (Rev). The 3' homology region }(I d h A) \text { was amplified with oCJ079 (Fwd), which incorportated the tac promoter, and oCJ080 } \\
\text { (Rev). These fragments were then assembled into pCM433 digested with XhoI and SacI. }\end{array}$ \\
\hline pCJ008 & $\begin{array}{l}\text { To replace } c a t B C A \text { with } \\
\text { Ptac:xylEGFJQKIH }\end{array}$ & $\begin{array}{l}\text { The 5' homology region was amplified from P. putida KT2440 genomic DNA with primers oCJ042 (Fwd), and oCJ043 } \\
\text { (Rev), which incorporated the tac promoter. The xylEGFJQKIH operon was amplified from P. putida mt-2 (ATCC 23973) } \\
\text { gDNA using primers oCJ044 (Fwd) and oCJ048 (Rev). The 3' homology region was amplified using primers oCJ046 and } \\
\text { oCJ047. These fragments were then assembled into pCM } 433 \text { digested with AatII and SacI. }\end{array}$ \\
\hline pCJ010 & To delete $a c e E F$ & $\begin{array}{l}\text { The 5' homology region was amplified from P. putida KT2440 genomic DNA with primers oCJ092 (Fwd) and oCJ093 } \\
\text { (Rev) and the 3' homology region was amplified using primers oCJ094 (Fwd) and oCJ095 (Rev). These fragments were } \\
\text { then assembled into pCM433 digested with AatII and SacI. }\end{array}$ \\
\hline pCJ011 & $\begin{array}{l}\text { To delete pcaHG and } \\
\text { used as an intermediate } \\
\text { in construction of } \\
\text { pCJ019 and pCJ0120 }\end{array}$ & $\begin{array}{l}\text { The 5' homology region was amplified from P. putida KT2440 genomic DNA with primers oCJ100 (Fwd) and oCJ101 } \\
\text { (Rev) and the 3' homology region was amplified using primers oCJ102 (Fwd) and oCJ103 (Rev). These fragments were } \\
\text { then assembled into pCM433 digested with AatII and SacI. }\end{array}$ \\
\hline pCJ012 & To delete $l l d D$ & $\begin{array}{l}\text { The 5' homology region was amplified from P. putida KT2440 genomic DNA with primers oCJ117 (Fwd) and oCJ118 } \\
\text { (Rev) and the 3' homology region was amplified using primers oCJ119 (Fwd) and oCJ120 (Rev). These fragments were } \\
\text { then assembled into pK18mobsacB digested with EcoRI and BamHI. }\end{array}$ \\
\hline pCJ015 & $\begin{array}{l}\text { To replace } \operatorname{ldhA} \text { with } \\
\text { Ptac:LDHA } A^{\text {B.t.N109G }}\end{array}$ & 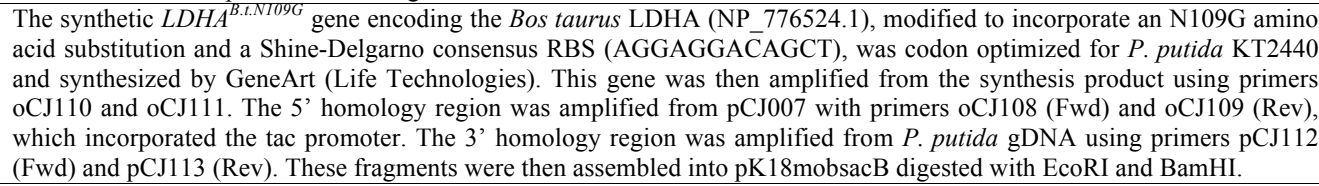 \\
\hline pCJ019 & $\begin{array}{l}\text { To replace } p c a H G \text { with } \\
\text { Ptac:ligABCIUJK }\end{array}$ & $\begin{array}{l}\text { The ligABCIUJK genes from Sphingobium sp. SYK-6 were codon optimized for } P \text {. putida KT2440 and synthesized by } \\
\text { GeneArt (Life Technologies) in two fragments (Table S4), ligABCI and ligUJK, in which Shine-Delgarno consensus RBSs } \\
\text { (AGGAGGACAGCT) were included 5' of the start codon of each gene. ligABCI and ligUJK were amplified from the } \\
\text { synthesized fragments with oCJ154 (Fwd), which incorporated the tac promoter, and oCJ157 (Rev) or oCJ158 (Fwd) and } \\
\text { oCJ155 (Rev), respectively. These fragments were then assembled in pCJ011 digested with BglII and NotI. }\end{array}$ \\
\hline pCJ120 & $\begin{array}{l}\text { To integrate the tac } \\
\text { promoter upstream of } \\
\text { pcaHG }\end{array}$ & $\begin{array}{l}\text { A portion of pcaHG was amplified from P. putida KT2440 genomic DNA using primers oCJ132 (Fwd), which incorporated } \\
\text { the tac promoter, and oCJ133 (Rev) and assembled into pCJ011 digested with BglII and SacI. }\end{array}$ \\
\hline
\end{tabular}


Table S2. Primers used in construction of gene replacement plasmids

\begin{tabular}{|c|c|}
\hline Primer & Sequence (5'-3') \\
\hline oCJ038 & CCGAAAAGTGCCACCTGACGTCCTTCATCGCCGGCCTG \\
\hline oCJ039 & GCCGCAGCTCGAGATCTGTCTTGTTCTGTTCGGTTCAGG \\
\hline оCJ040 & AGATCTCGAGCTGCGGCCGCTCCACCGAGTGGGCTG \\
\hline oCJ041 & GCTGGATCCTCTAGTGAGCTCGGTTTTCATGGGCTTCATGGC \\
\hline oCJ042 & CCGAAAAGTGCCACCTGACGTCCCTGTTGCTCGATCAACGC \\
\hline oCJ043 & TCATAAGATCTCTCCTGTGTGAAATTGTTATCCGCTCACAATTCCACACATTATACGAGCCGATGATTAATTGTCAACAGCTCTGTTGCCAGGTCCCGTC \\
\hline oCJ044 & AGGAGAGATCTTATGAACAAAGGTGTAATGCGACC \\
\hline oCJ046 & ATTGCGCGGCCGCGTTCGAGGTTATGTCACTGTGATTTTG \\
\hline oCJ047 & GCTGGATCCTCTAGTGAGCTCCGCCTGCTCCAGGTTG \\
\hline oCJ048 & CGAACGCGGCCGCGCAATTCAGCGTCTGACCTTGCTG \\
\hline oCJ049 & AGGAGAGATCTTATGACAAGCGTGCTGATTGAACAC \\
\hline oCJ050 & GCTGGATCCTCTAGTGAGCTCTTGATTGCTGATCAGCGACG \\
\hline oCJ077 & GATGCATATGCTGCAGCTCGAGCGTTACCACTGC \\
\hline oCJ078 & GCTCGCGGCCGCTGCCTGTCAAGGCATGC \\
\hline оСJ079 & $\begin{array}{l}\text { AGGCAGCGGCCGCGAGCTGTTGACAATTAATCATCGGCTCGTATAATGTGTGGAATTGTGAGCGGATAACAATTTCACACAGGAGGACAGCTATGACCCACC } \\
\text { CAAGACATGC }\end{array}$ \\
\hline oCJ080 & GGCTGGATCCTCTAGTGAGCTCGGGTCCTCCAGAAATGC \\
\hline oCJ100 & CCGAAAAGTGCCACCTGACGTCGGCCTTGCTGCTGCAG \\
\hline oCJ101 & GCCGCAGCTCGAGATCTGGAATTGTGAGAACGCCTGG \\
\hline oCJ102 & AGATCTCGAGCTGCGGCCGCGGTGAAGCTTGGGGCC \\
\hline oCJ103 & GCTGGATCCTCTAGTGAGCTCACGATTTCCCCATTGCCAG \\
\hline oCJ108 & CAGCTATGACATGATTACGAATTCCTCGAGCGTTACCACTGC \\
\hline oCJ109 & GCTGTCCTCCTGTGTGAAATTG \\
\hline oCJ110 & CAATTTCACACAGGAGGACAGC \\
\hline oCJ111 & GAAATGCCGCGTATCCTAGC \\
\hline oCJ112 & GCTAGGATACGCGGCATTTC \\
\hline oCJ113 & СTGCAGGTCGACTCTAGAGGATCCCATCGACGACAAGGGCAAG \\
\hline oCJ117 & CAGCTATGACATGATTACGAATTCTCGGCCCGGAACTGC \\
\hline oCJ118 & AGCGTTGCTTGTGGGAGTG \\
\hline oCJ119 & САCTCCCACAAGCAACGCTACGCCTGCAAGAACCAC \\
\hline oCJ120 & CTGCAGGTCGACTCTAGAGGATCCGCACCGAGCGCAGG \\
\hline oCJ132 & $\begin{array}{l}\text { GGCGTTCTCACAATTCCAGATCTGAGCTGTTGACAATTAATCATCGGCTCGTATAATGTGTGGAATTGTGAGCGGATAACAATTTCACACAGGAGGACAGCT } \\
\text { ATGCCCGCCCAGGA }\end{array}$ \\
\hline oCJ133 & GGCTGGATCCTCTAGTGAGCTCGAAGGCGTTTTCCAGGTTGTAG \\
\hline oCJ154 & $\begin{array}{l}\text { GGCGTTCTCACAATTCCAGATCTGAGCTGTTGACAATTAATCATCGGCTCGTATAATGTGTGGAATTGTGAGCGGATAACAATTTCACACAGGAGGACAGCT } \\
\text { ATGACCGAGAAGAAAGAACGCATCG }\end{array}$ \\
\hline oCJ155 & GCGGCCCCAAGCTTCACCGCGGCCGCTCAGACGTACTTCAGGCCCTC \\
\hline oCJ157 & TCACATTTCCTCCGACCAGTACAG \\
\hline oCJ158 & ACTGGTCGGAGGAAATGTGAAGGAGGACAGCTATGCCAGGC \\
\hline
\end{tabular}

Table S3. Primers used to confirm gene replacements by colony PCR

\begin{tabular}{|c|c|c|c|c|}
\hline Function & Product & Correct size (bp) & Primer & Sequence (5'-3') \\
\hline \multirow{3}{*}{$\begin{array}{l}\text { To confirm integration of the } \\
\text { tac promoter upstream of } \\
\text { catBCA }\end{array}$} & 5' junction & 1,076 & Fwd: oCJ086 & TGTGGGCATGGTGTGTTC \\
\hline & \multirow[t]{2}{*}{ 3' junction } & \multirow[t]{2}{*}{1,233} & Fwd: oCJ054 & ATCGGCTCGTATAATGTGTGG \\
\hline & & & Rev: oCJ090 & GCTTCACGGTCATCTTCAC \\
\hline \multirow{3}{*}{$\begin{array}{l}\text { To confirm replacement of } \\
\text { catBCA with } x y l E G F J Q K I H\end{array}$} & 5' junction & 2,098 & Rev: oCJ091 & ACGAAGGCACCGCTAATG \\
\hline & \multirow[t]{2}{*}{ 3' junction } & \multirow[t]{2}{*}{1,329} & Fwd: oCJ070 & AACATCACCGTGCGCTAC \\
\hline & & & Rev: oCJ087 & TCTTCAAAGCGTCCGGTG \\
\hline $\begin{array}{l}\text { To confirm integration of the } \\
\text { tac promoter upstream of } \\
\text { pcaHG }\end{array}$ & 3' junction & 1,182 & Rev: oCJ135 & AGGCTGATGTTGATGTGC \\
\hline \multirow{4}{*}{$\begin{array}{l}\text { To confirm replacement of } \\
\text { pcaHG with ligABCIUJK }\end{array}$} & \multirow[t]{2}{*}{ 5' junction } & \multirow[t]{2}{*}{1,069} & Fwd: oCJ106 & АTCTTGAACCAACGCACC \\
\hline & & & Rev: oCJ055 & TCCGCTCACAATTCCACAC \\
\hline & \multirow[t]{2}{*}{ 3' junction } & \multirow[t]{2}{*}{1,288} & Fwd: oCJ153 & ACCGTGAAGAACACCCTG \\
\hline & & & Rev: oCJ107 & CACAAGGCAATCCTGATCG \\
\hline To confirm deletion of catA2 & Entire locus & 2,089 & Fwd: oCJ084 & ССTCAATGGCTTTGCCAG \\
\hline \multirow{4}{*}{$\begin{array}{l}\text { To confirm replacement of } \\
l d h A \text { with Ptac: } L D H A^{B . t . N I 09 G}\end{array}$} & \multirow[t]{2}{*}{$5^{\prime}$ junction } & \multirow[t]{2}{*}{1,485} & Fwd: oCJ088 & GGGCCATTATCATCAGCAG \\
\hline & & & Rev: oCJ130 & TAGGTCAGGATGTCCACCG \\
\hline & \multirow[t]{2}{*}{ 3' junction } & 1,667 & Fwd: oCJ129 & TGGACATCCTGACCTACG \\
\hline & & & Rev: oCJ131 & GTATCGGTTTCACCGTGC \\
\hline
\end{tabular}


Table S4. Sequence of synthetic DNA fragments containing the ligABCIUJK genes from Sphingobium sp. SYK-6 optimized for expression in P. putida K2440. The ORFs are shown in upper case and start and stop codons are indicated by bold text.

\section{Fragment Sequence (5'-3')}

$\operatorname{lig} A B C I$

aggaggacagctATGACCGAGAAGAAAGAACGCATCGACGTGCACGCCTACCTGGCCGAGTTCGACGACATCCCAGGCACCCGTGTGTTCACCGCCCAGCGT GCCCGTAAGGGCTACAACCTGAACCAGTTCGCCATGAGCCTGATGAAGGCCGAGAACCGCGAGCGCTTCAAGGCCGACGAGAGCGCCTACCTGGACGAATGG AACCTGACCCCAGCCGCCAAAGCCGCCGTGCTGGCCCGTGACTACAACGCCATGATCGACGAGGGTGGCAACGTGTACTTCCTGAGCAAGCTGTTCAGCACC GACGGCAAGAGCTTCCAGTTCGCCGCCGGTAGCATGACCGGCATGACCCAAGAGGAATACGCCCAGATGATGATCGATGGCGGTCGCAGCCCAGCCGGTGTG CGCAGCATCAAGGGTGGCTACTGAaggaggacagctATGGCCCGTGTGACCACCGGCATCACCAGCAGCCACATCCCAGCCCTGGGTGCCGCCATCCAAACC GGCACCAGCGACAACGACTACTGGGGTCCGGTGTTCAAGGGCTACCAGCCGATCCGCGACTGGATCAAGCAGCCAGGCAACATGCCGGACGTGGTGATCCTG GTGTACAACGACCACGCCAGCGCCTTCGACATGAACATCATCCCGACCTTCGCCATCGGCTGCGCCGAAACCTTCAAGCCAGCCGACGAGGGCTGGGGTCCG CGTCCAGTGCCGGATGTGAAGGGCCATCCGGACCTGGCCTGGCATATCGCCCAGAGCCTGATCCTGGACGAATTCGATATGACCATCATGAACCAGATGGAC GTGGACCACGGCTGCACCGTGCCGCTGAGCATGATCTTCGGCGAGCCGGAAGAGTGGCCGTGCAAGGTGATCCCGTTCCCGGTGAACGTGGTGACCTATCCG CCACCGAGCGGCAAGCGCTGCTTCGCCCTGGGCGACAGCATCCGTGCCGCCGTGGAAAGCTTCCCCGAGGACCTGAACGTGCACGTGTGGGGCACCGGTGGC ATGTCGCACCAGCTGCAAGGTCCGCGTGCCGGTCTGATCAACAAAGAGTTCGACCTGAACTTCATCGACAAGCTGATCAGCGACCCGGAAGAACTGAGCAAG ATGCCGCACATCCAGTACCTGCGCGAGAGCGGCAGCGAGGGCGTGGAACTGGTGATGTGGCTGATCATGCGTGGTGCCCTGCCGGAAAAGGTGCGCGACCTG TACACCTTCTACCATATCCCAGCCAGCAACACCGCGCTGGGTGCCATGATCCTGCAGCCGGAAGAAACCGCCGGCACCCCACTGGAACCGCGTAAGGTGATG AGCGGTCACAGCCTGGCCCAGGCCTGAaggaggacagctATGCGTATCGCCCTGGCCGGTGCCGGTGCCTTCGGCGAAAAGCATCTGGACGGCCTGAAGAAC ATCGACGGCGTGGAAATCGTGAGCATCATCAGCCGCAAGGCCGAGCAAGCCGCCGAGGTGGCCGCCAAGTACGGTGCCAAACACAGCGGCACCGACCTGAGC GAAGCCCTGGCCCGTGATGACGTGGACGCCGTGATCCTGTGCACCCCGACCCAGATGCACGCCGAGCAAGCGATCGCCTGCATGAACGCCGGTAAGCACGTG CAGGTCGAGATCCCGCTGGCCGACAGCTGGGCCGACGCCGAGGCCGTGATGAAGAAGTCGCAAGAAACCGGTCTGGTGTGCATGGTGGGCCACACCCGTCGC TTCAACCCGAGCCACCAGTACATCCACAACAAGATCGTGGCCGGTGAGCTGGCCATCCAGCAGATGGACGTCCAGACCTACTTCTTCCGTCGCAAGAACATG AACGCCAAGGGCGAACCGCGTAGCTGGACCGACCATCTGCTGTGGCACCATGCCGCCCACACCGTGGACCTGTTCGCCTACCAAGCCGGTAAGATCGTCCAG GCCAACGCCGTGCAGGGTCCGATCCACCCGGAACTGGGTATCGCCATGGACATGAGCATCCAGCTGAAGTCGGAAACCGGTGCCATCTGCACCCTGAGCCTG AGCTTCAACAACGACGGTCCGCTGGGCACCTTCTTCCGCTACATCTGCGACAACGGCACCTGGATCGCCCGTTACGACGACCTGGTGACCGGCAAAGAGGAA CCGGTCGACGTCAGCAAGGTGGACGTGAGCATGAACGGCATCGAGCTGCAGGACCGCGAGTTCATCGCCGCCATCCGCGAAGGCCGTGAGCCGAACAGCAGC GTGGCCCGTGTGCTGGACTGCTACCGCGTGCTGGGCGAGCTGGAAGTGCAGCTGGAAAAGCAGGGCTGAaggaggaCagCtATGACCAACGACGAGCGCATC CTGAGCTGGAACGAAACCCCGAGCAAGCCACGCTACACCCCACCGCCAGGTGCCATCGACGCCCACTGCCATGTGTTCGGTCCGATGGCCCAGTTCCCGTTC AGCCCGAAGGCCAAGTACCTGCCACGTGATGCCGGTCCGGACATGCTGTTCGCGCTGCGCGACCATCTGGGCTTCGCCCGTAACGTGATCGTGCAGGCCAGC TGCCACGGCACCGATAACGCCGCCACCCTGGATGCGATCGCCCGTGCCCAAGGCAAAGCCCGTGGTATCGCCGTGGTGGACCCAGCCATCGACGAGGCCGAA CTGGCCGCCCTGCACGAAGGCGGTATGCGTGGTATCCGCTTCAACTTCCTGAAGCGCCTGGTGGACGATGCCCCAAAGGACAAGTTCCTGGAAGTGGCCGGT CGCCTGCCAGCCGGTTGGCACGTGGTGATCTACTTCGAGGCCGACATCCTGGAAGAACTGCGTCCGTTCATGGACGCCATCCCGGTGCCGATCGTGATCGAC CACATGGGTCGTCCGGACGTGCGCCAGGGTCCAGACGGTGCCGACATGAAGGCCTTCCGTCGCCTGCTGGACAGCCGTGAGGACATCTGGTTCAAGGCGACC TGCCCAGACCGCCTGGACCCAGCCGGTCCGCCATGGGATGATTTCGCCCGTAGCGTGGCCCCACTGGTGGCCGATTATGCCGACCGCGTGATCTGGGGCACC GACTGGCCACACCCGAACATGCAGGACGCGATCCCGGACGACGGCCTGGTGGTGGACATGATCCCACGTATCGCCCCAACCCCAGAGCTGCAGCACAAGATG CTGGTGACCAACCCGATGCGCCTGTACTGGTCGGAGGAAATGTGA

ligUJK aggaggacagctATGCCAGGCGGTGTGCGCTGCATGTGGATGCGTGGTGGCACCAGCAAAGGCGGTTACTTCCTGGCCGAGGACCTGCCAGCCGACACCGCG ACCCGTGATGCCTTCCTGCTGCGCGTGATGGGCAGCCCAGACCCACGCCAGATCGACGGCATGGGTGGTGCCGATCCGCTGACCTCGAAGGTGGCCGTGGTG AAGAAAAGCGCCCGTGAGGGCGTGGACGTGGACTACCTGTTCCTGCAAGTGTTCGTGGACCAGGCCATCGTGACCGACGCCCAGAACTGCGGCAACATCCTG GCCGGTATCGGTCCGTTCGCCATCGAGCGTGGTCTGGTGGCCCCAACCGGTGACGAAACCCGTGTGGCCATCTTCATGGAAAACACCGGTCAGATCGCCGTG GCCACCGTGCAGACCCCAGGTGGCACCGTGACCTACGATGGCGAAGCCGCCATCACCGGTGTGCCAGGCACCGCCGCCCCAGTGCCGCTGCTGTTCCGCGAC ACCGCCGGTAGCTCGTGCGGTGCCCTGCTGCCAACCGGCAACGGCGTGGACGAAATCGACGGCGTCCGCGTGACCATGATCGACAACGGCATGCCCTGCGTG GTGATGCTGGCCAGCGACGTGGGCGTGACCGGCTACGAAGATCGCGACACCCTGGACGCCAACGCCGAGATGAAGGCCAAGGTGGAAGCCATCCGCCTGAAG GTCGGTGCCATGATGAACCTGGGCGACGTGACCGAGAAGTCGGTGCCGAAGATGATGCTGGTCGCCCCACCGCGTGAAGGCGGTGCGATCACCGTGCGTAGC CTGATCCCGCACCGCGTGCATGCCAGCATCGGTGTGCTGGGTGCCGTGAGCGTGGCCACCGCCTGCCTGATCGAAGGCTCGCCAGCCGCCAGCGTCGCCACC GTGCCAGACGGTGCCACCAAAACCCTGGGCGTGGAACACCCGACCGGTGTGACCGAGTGCGTGGTGACCGTGGATGCCGCCGGTCAACCGGTGGAAGCCGGT ATGCTGCGCACCGCCCGTAAGCTGATGGACGGCATCGTGTTCGGCTGAaggaggacagctATGATGATGATCATCGACTGCCACGGCCACTACACCGTGCTG CCGAAGGCCCACGACGAGTGGCGTGAGCAGCAGAAAGCCGCCTTCAAAGCCGGTCAGCCAGCCCCACCGTACCCGGAAATCAGCGACGACGAGATCCGCGAA ACCATCGAGGCCAACCAGCTGCGCCTGATCAAAGAACGCGGTGCGGACATGACCATCTTCAGCCCACGTGCCAGCGCCATGGCCCCACATGTCGGCGATCAA AGCGTGGCCGTGCCGTGGGCCCAGGCGTGCAACAACCTGATCGCCCGTGTGGTCGACCTGTTCCCGGAAACCTTCGCCGGTGTGTGCATGCTGCCGCAGAGC CCAGAGGCCGACATGACCAGCTCGATCGCCGAGCTGGAACGCTGCGTGAACGAGCTGGGCTTCATCGGCTGCAACCTGAACCCGGACCCAGGCGGTGGTCAC TTCAAGCACCCACCGCTGACCGACCGTTTCTGGTATCCGTTCTACGAGAAGATGGTGGAACTGGACGTGCCAGCCATGATCCACGTGAGCGGCAGCTGCAAC CCAGCCATGCATGCGACCGGTGCCTACTACCTGGCCGCCGACACCATCGCCTTCATGCAGCTGCTGCAGGGCAACCTGTTCGCCGACTTCCCGACCCTGCGC TTCATCATCCCGCACGGTGGTGGTGCCGTGCCGTACCACTGGGGTCGTTTCCGTGGCCTGGCCGACATGCTGAAGCAGCCGAGCCTGGACACCCTGCTGATG AACAACGTGTTCTTCGATACCTGCGTGTACCATCAGCCAGGCATCAACCTGCTGGCCGACGTCATCGACAACAAGAACATCCTGTTCGGCAGCGAGATGGTG GGTGCCGTGCGTGGCATCGACCCGACCACCGGTCACTACTTCGACGACACCAAGCGCTACATCGACGCCCTGGACATCAGCGACCAAGAGCGCCACGCCATC TTCGAGGGCAACACCCGTCGTGTGTTCCCACGTCTGGACGCCAAGCTGAAAGCCCGTGGCCTGTGAaggaggacagCtATGCGTGGTGCCGCCATGGGCGTG GTGGTGCAGAACATCGAGCGTGCGCCACTGGAAGTGATCGACGGCCTGGCCGCCTGCGGTGTGGCCACCGTCCACGAAGCCCAAGGCCGTACCGGTCTGCTG GCCAGCTACATGCGTCCGATCTATCGCGGTGCCCGTGTGGCCGGTAGCGCCCTGACCATCAGCGCCCCACCGGGTGACAACTGGATGGTGCACGTGGCCATC GAGCAGCTGAAAGCGGGTGACATCCTGCTGCTGGCCCCAACCAGCCCGTGCGAGGATGGCTACTTCGGCGACCTGCTGGCGACCAGCGCCCAAGCCCGTGGC TGCCGTGGTCTGGTCATCGATGCCGGTGTGCGTGACGTCCGCGACCTGACCGAGATGAACTTCCCGGTGTGGTCGAAGGCCATCTACGCCCAGGGCACCGTG AAGAACACCCTGGGCAGCGTGAACGTGCCGGTGGTGTGCGCCAACGCCCTGGTGAACCCAGGCGACGTGATCGTGGCCGACGACGACGGTGTGTGCGTGGTC CCGCTGGCCAACGCCGAAAAGGTGCTGGAAGCCGCCCGTGCCCGTGAGGCCAACGAAGGCGACAAGCGCGAGAAAATGGCCAACGGCGTGCTGGGCCTGGAC CTGTACAAGATGCGCGAGCGCCTGGAAAAAGAGGGCCTGAAGTACGTCTGA 


\section{RESULTS}
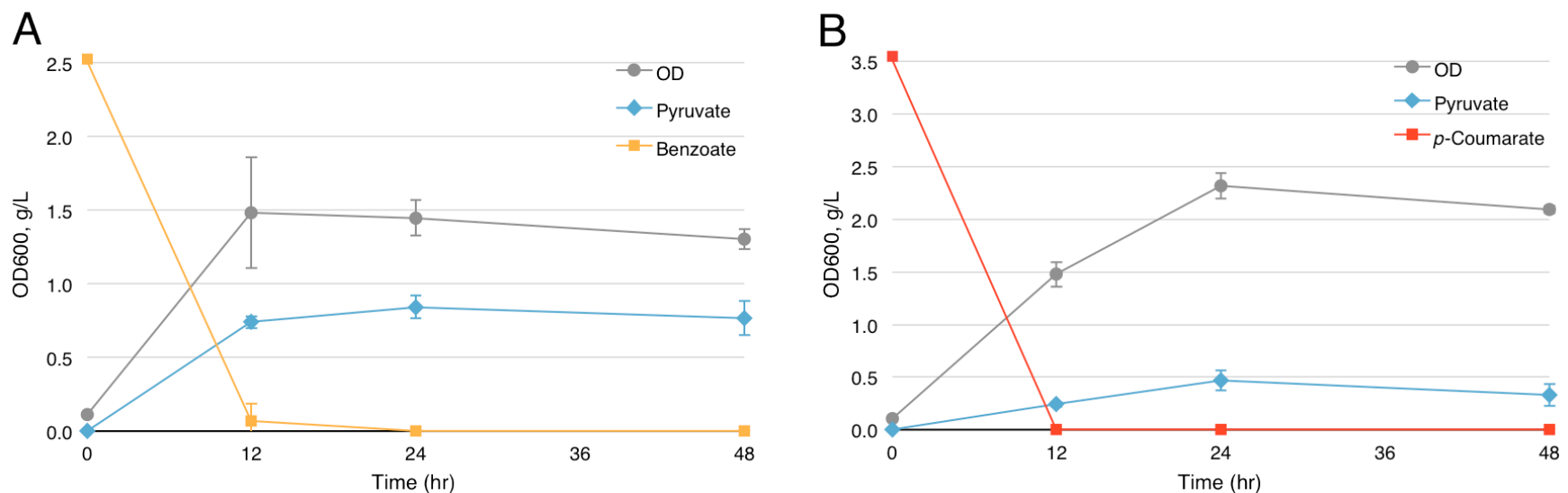

Fig S1. Production of pyruvate from benzoate by engineered $P$. putida strain CJ051 ( $\triangle a c e E F)$. Strains were grown in M9 minimal media containing either benzoate or $p$-coumarate as the sole carbon and energy source and sampled periodically to evaluate culture growth by OD600 and measure benzoate and pyruvate content by HPLC. Each line represents the average of three biological replicates. The error bars represent standard deviation of the measurements. (A) CJ051 grown on media containing $20 \mathrm{mM}$ benzoate. (B) CJ051 grown on media containing $20 \mathrm{mM} p$-coumarate.

Table S5. Pyruvate yields from engineered strains. Values represent the average and standard deviation of biological triplicates.

\begin{tabular}{lllcc}
\hline Strain & Degradation pathway & Genotype & Substrate & $\begin{array}{c}\text { Pyruvate } \\
\text { \% yield (wt/wt) }\end{array}$ \\
\hline CJ051 & Catechol ortho & $\Delta a c e E F$ & benzoate & $23.9 \pm 3.1$ \\
CJ113 & Catechol ortho & $\Delta a c e E F$ Ptac:catBCA & benzoate & $30.7 \pm 4.6$ \\
CJ051 & Protocatechuate ortho & $\Delta a c e E F$ & p-coumarate & $9.3 \pm 2.9$ \\
CJ116 & Protocatechuate ortho & $\Delta a c e E F$ Ptac:pcaHG & p-coumarate & $12.1 \pm 3.9$ \\
\hline
\end{tabular}

